Check for updates

Cite this: Phys. Chem. Chem. Phys., 2021, 23, 3106

Received 21st September 2020, Accepted 17th January 2021

DOI: $10.1039 / \mathrm{d} 0 \mathrm{cp} 04985 \mathrm{a}$

rsc.li/pccp

\section{Diffusion of the carbon dioxide-ethanol mixture in the extended critical region $\dagger$}

\author{
René Spencer Chatwell, (D) a Gabriela Guevara-Carrion, (D) a Yuri Gaponenko, \\ Valentina Shevtsova (D) ${ }^{b}$ and Jadran Vrabec (D)*a
}

\begin{abstract}
The effect of traces of ethanol in supercritical carbon dioxide on the mixture's thermodynamic properties is studied by molecular simulations and Taylor dispersion measurements. This mixture is investigated along the isobar $p=10 \mathrm{MPa}$ in the temperature range between $T=304$ and $343 \mathrm{~K}$. Along this path, the mixture undergoes two transitions: First, the Widom line is crossed, marking the transition from liquid-like to gas-like conditions. A second transition occurs from the supercritical gas-like domain to a subcritical gas. The Widom line crossover entails inflection points for most of the studied properties, i.e. density, enthalpy, shear viscosity, Maxwell-Stefan and intradiffusion coefficients. On the other hand, the transition between the super- and subcritical regions is found to be generally smooth, an observation that is qualitatively confirmed by experimental Taylor dispersion measurements. Dedicated atomistic simulations show the presence of microheterogeneities due to ethanol self-association along the investigated path, which lead to the mixture's anomalous behavior in its extended critical region.
\end{abstract}

\section{Introduction}

Investigating the thermophysical properties of supercritical carbon dioxide $\left(\mathrm{scCO}_{2}\right)$ has grown to a topic of great interest. ${ }^{1-4}$ While the majority of work had focused on pure fluids in sufficient distance from the critical point, ${ }^{5,6}$ only some special case scenarios with respect to a second component have been examined under near-critical conditions. ${ }^{7-9}$

$\mathrm{CO}_{2}$ is commonly used in various technological applications, ranging from environmental, mechanical, chemical, geothermal to pharmaceutical industries. ${ }^{10}$ It constitutes an attractive alternative to organic or aqueous solvents by having a remarkably mild critical point, being non-toxic, non-flammable, widely available and largely inert. However, due to its low dielectric constant in combination with a zero dipole moment, ${ }^{11}$ it is a poor solvent for polar substances. Yet, the solubility of high molecular weight solutes is readily enhanced by adding small

\footnotetext{
${ }^{a}$ Thermodynamics and Process Engineering, Technische Universität Berlin, 10587 Berlin, Germany. E-mail: vrabec@tu-berlin.de; Tel: +49 3031422755

${ }^{b}$ Microgravity Research Center, Université Libre de Bruxelles, 1050 Bruxelles, Belgium

$\dagger$ Electronic supplementary information (ESI) available: It includes a schematic of the employed Taylor dispersion apparatus, graphical results for the enthalpy $h$, specific volume $v, c_{\mathrm{p}}, c_{\mathrm{v}}, \alpha_{\mathrm{v}}$ and $\beta_{\mathrm{T}}$, as well as the center-of-mass radial distribution function and average coordination number of the $\mathrm{CO}_{2}$-ethanol pair. Predictions of the Fick diffusion coefficient of the $\mathrm{CO}_{2}+$ ethanol mixture with the regarded equations are shown in comparison with experimental and simulation results. The numerical data from equilibrium molecular dynamics simulation are also listed. See DOI: 10.1039/d0cp04985a
}

amounts of strongly polar entrainers. ${ }^{12,13}$ Consequently, ethanol emerges as a widespread commodity in supercritical extraction processes. Further, ethanol forms a non-ideal mixture with $\mathrm{CO}_{2}$ due to the presence of strong solute-solute interactions and hydrogen bonding making this mixture particularly interesting.

In contrast to the classical perception of the supercritical region as a featureless domain, experimental evidence ${ }^{14-17}$ and molecular dynamics simulations ${ }^{3,4,9,18-20}$ indicate a partition into several subdomains. The continuous dynamic crossover between different domains occurs across transition lines, i.e. Fisher-Widom, ${ }^{21}$ Nishikawa, ${ }^{14,16}$ Frenkel $^{22,23}$ and Widom. ${ }^{24}$ For moderate pressures, i.e. up to three times the critical pressure ${ }^{25} p \leq 3 p_{c}$, the crossover between gas-like and liquidlike regimes is accomplished over a delta shaped area ${ }^{20,26,27}$ that is delimited by the loci of extrema of particular thermodynamic response functions, i.e. isobaric and isochoric heat capacities $c_{\mathrm{p}}, c_{\mathrm{v}}$, thermal expansion $\alpha_{\mathrm{v}}$, isothermal compressibility $\beta_{\mathrm{T}}$, density $\rho$ and speed of sound $c$. These response functions eventually confluence to a single line in close vicinity to the critical point, the so-called Widom line, which can be considered as an extension of the vapor pressure curve. Crossing the Widom line is additionally associated with a minimum of the thermodynamic factor and with large density fluctuations. ${ }^{28}$

While the critical point exhibits critical opalescence, ${ }^{29}$ its extended vicinity is characterized by emerging microscopic clusters that have been observed experimentally ${ }^{28}$ and with equilibrium molecular dynamics simulations. ${ }^{30-34}$ The present work contributes to the understanding of how ethanol diluted into $\mathrm{CO}_{2}$ affects the mixture's microscopic structure and 
consequently its transport properties in the extended critical region. The $\mathrm{CO}_{2}+$ ethanol mixture was examined twofold. While the microscopic structure was investigated exclusively by equilibrium molecular dynamics (EMD) simulations, mutual diffusion was sampled atomistically and was measured in the lab with the Taylor dispersion technique. The present mixture has already been investigated experimentally ${ }^{35-37}$ and by molecular simulations. In particular, the vapor-liquid equilibrium (VLE), ${ }^{38}$ solubility parameters, ${ }^{39}$ microscopic structure $^{31,32,40-42}$ and diffusion coefficients ${ }^{43}$ have been studied by molecular simulation. However, the effects of structural changes on mutual diffusion across the Widom line in the extended critical region of this mixture have not yet been considered neither experimentally, nor by molecular simulation. This is of special interest for the understanding and description of supercritical extraction processes which are performed in this region.

\section{Method}

\subsection{Molecular simulation}

Classical molecular simulations are based on molecular force fields models that are typically optimized to describe the pure component phase behavior. The transferability to a given mixture is validated by comparing simulation predictions to experimental data along the VLE curves. While the employed force fields were based on the Lennard-Jones potential for both components, a predictive mode was used to describe the interactions between unlike Lennard-Jones sites, i.e. the Lorentz-Berthelot combining rules were assumed. The force field for ethanol consisted of three Lennard-Jones sites with three superimposed point charges. ${ }^{44}$ In contrast, the two Lennard-Jones sites of carbon dioxide's force field included a superimposed point quadrupole. ${ }^{45}$ States along the VLE curves were sampled by Monte Carlo (MC) simulations with the grand equilibrium method containing $N=1372$ molecules for the liquid phase and $N=460$ molecules for the gas phase simulations.

The MC simulation results for the VLE curves are in excellent agreement with experimental literature data ${ }^{46-52}$ over the entire investigated temperature and pressure ranges, $c f$. Fig. 1 . In order to properly appreciate the simulation results, the coexistence curves were additionally computed by three equations of state, allowing for a temperature-dependent binary parameter $k_{i j}$, $c f$. Table 1. While the employed cubic equations of state, i.e. SoaveRedlich-Kwong ${ }^{53}$ and Peng-Robinson, ${ }^{54}$ tend to overestimate the mixture's vapor pressure at high $\mathrm{CO}_{2}$ mole fractions, the $\mathrm{PCP}^{\mathrm{SAFT}}{ }^{55}$ equation of state overestimates the vapor pressure at low $\mathrm{CO}_{2}$ mole fractions.

The intra- $D_{i}$ and Maxwell-Stefan $D$ diffusion coefficients as well as the shear viscosity were sampled directly with EMD simulation and the Green-Kubo formalism. ${ }^{57,58}$ The working equations for the determination of these transport properties have been published e.g. in ref. 59 and are not repeated here. EMD simulations for transport properties were made in two steps. In the first step, a simulation in the

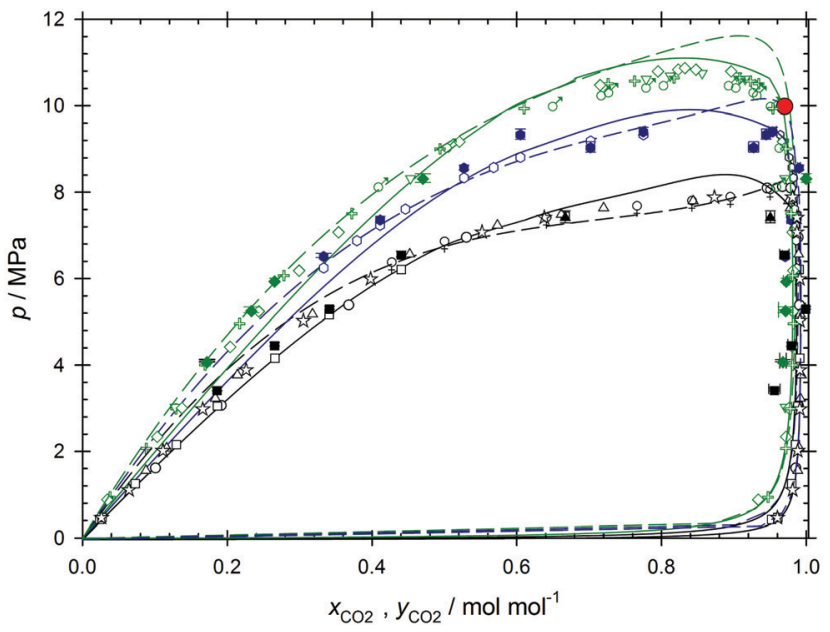

Fig. 1 Vapor-liquid phase diagram of $\mathrm{CO}_{2}+$ ethanol. The open symbols indicate experimental literature data along three isotherms depicted in black $T=312 \mathrm{~K}$ (bullets, ${ }^{49}$ squares, ${ }^{49}$ triangles, ${ }^{50}$ stars, ${ }^{46}$ male symbols ${ }^{47}$ ), blue $T=325 \mathrm{~K}$ (hexagons ${ }^{56}$ ) and green $T=333 \mathrm{~K}$ (diamonds, ${ }^{49}$ inverted triangles, ${ }^{49}$ crosses $^{46}$ female symbols ${ }^{47}$ ). The solid symbols are present molecular simulation results. The solid lines represent the binodals according to the Peng-Robinson and the dashed lines according to the PCP-SAFT equation of state. The solution according to the Soave-Redlich-Kwong equation was omitted to maintain readability. The red bullet symbolizes the molar composition $x_{\mathrm{CO} 2}=0.97 \mathrm{~mol} \mathrm{~mol}^{-1}$ and pressure $p=10 \mathrm{MPa}$ at which all other molecular dynamics simulations were carried out.

Table 1 Each of the employed equations of state was fitted to experimental VLE data, resulting in a linear temperature dependence of the binary interaction parameter, i.e. $k_{i j}=m(T / K-305)+C$

\begin{tabular}{lll}
\hline Equation of state & $m / 10^{-4}$ & $C$ \\
\hline Peng-Robinson & -1.7 & 0.0879 \\
Soave-Redlich-Kwong & -1.0 & 0.0863 \\
PCP-SAFT & -3.0 & 0.0506
\end{tabular}

isobaric-isothermal $(N p T)$ ensemble was performed to calculate the density at the desired temperature and pressure. In the second step, EMD simulations were performed at this temperature and density in the canonical $(N V T)$ ensemble containing $N=3000$ molecules in cubic volumes with periodic boundary conditions and specifying an integrator time step of $\Delta t=1 \mathrm{fs}$. The associated finite size effects were corrected with a modified Yeh-Hummer approach ${ }^{60,61}$ employing the sampled shear viscosity values. To improve statistics, a total of $2.5 \times 10^{5}$ correlation functions was averaged. The thermodynamic factor $\Gamma$ was sampled directly with Kirkwood-Buff integration based on the methodology proposed by Ganguly and van der Vegt, ${ }^{62}$ which was found to be the most adequate in previous work. ${ }^{63}$ Extrapolation to the thermodynamic limit was not necessary. All molecular simulations were performed with the fully open source software $m s 2 .{ }^{64}$ Statistical uncertainties were calculated with the error propagation law and a coverage factor of $k=1$. Throughout, the cut-off radius was set to $r_{\mathrm{c}}=17.5 \AA$. Electrostatic long-range corrections were made using the 
reaction field technique with conducting boundary conditions $\left(\varepsilon_{\mathrm{RF}}=\infty\right)$.

\subsection{Taylor dispersion technique}

The employed Taylor apparatus has been optimized for the use of $\mathrm{ScCO}_{2}$ as described in preceding works. ${ }^{35,65,66}$ The experimental determination of the mixture's mutual diffusion coefficient $D$ constitutes a four stage procedure utilizing the Taylor dispersion technique. The experimental schematic is disclosed in the ESI. $\dagger$ During the first step, the pure $\mathrm{CO}_{2}$ carrier fluid which was stored under VLE conditions, i.e. at $T=288.15 \mathrm{~K}, p \sim 5 \mathrm{MPa}$ with a purity $0.99998 \mathrm{~mol} \mathrm{~mol}^{-1}$ (Air Liquide), was initially liquefied through a cryostat reducing its temperature to $T=269.15 \mathrm{~K}$. Liquid $\mathrm{CO}_{2}$ was subsequently pumped above the critical pressure and eventually heated to its target temperature before it reached the injection valve (Knauer model D-14163). In the second step, $\mathrm{scCO}_{2}$ was delivered to the dispersion tube with a constant flow rate. The carrier stream was thermostated by means of a heat reservoir ensuring a constant target temperature ranging from $T_{\exp }=304$ to $343 \mathrm{~K}$ with an accuracy of $\pm 0.1 \mathrm{~K}$ and barostated with a high pressure pump at $p_{\exp }=10 \mathrm{MPa}$ with $\pm 0.05 \mathrm{MPa}$ accuracy. The ethanol sample (purity 99.9\% (GC) in volume fraction, CAS 64-17-5; purchased from VWR) adopts to the respective target temperature $T_{\exp }$ within the valve's loop prior to its injection into the $\mathrm{scCO}_{2}$ stream. In order to ensure temperature homogenization in this section of the experiment, the dispersion tube and injection valve were placed inside a polyurethane foam insulated housing with an additional air fan. In the third step, the sample was injected into the $\mathrm{scCO}_{2}$ stream. The resulting strongly diluted mixture was fed to a $l=$ $30.916 \pm 0.001 \mathrm{~m}$ long dispersion capillary with a circular cross section of radius $r=0.375 \mathrm{~mm}$. The capillary was coiled around a grooved, hollow aluminum cylinder with radius $R_{\mathrm{c}}=0.175 \mathrm{~m}$ providing stability and fixation. The cylinder was additionally thermostated with an internal circular flow ensuring a temperature stabilization of $\pm 0.1 \mathrm{~K}$. In order to minimize pressure and density disturbances during injection, an ethanol sample volume of $V_{0}=2 \times 10^{-6} \mathrm{dm}^{3}$ was selected, with smaller volumes having a negative effect on the signal-to-noise ratio. ${ }^{35,65}$ The pressure of the system was controlled by a back pressure regulator (Jasco BP2080) and measured by means of a pressure sensor (JUMO dTrans p30) with an accuracy of $\pm 0.05 \mathrm{MPa}$. In the experiment's final step, the Taylor peak was monitored at the outlet of the dispersion tube by means of a FT-IR spectrometer (Jasco FT-IR 4100) with $\pm 0.01 \mathrm{~cm}^{-1}$ accuracy and $4 \mathrm{~cm}^{-1}$ resolution. In contrast to its nominal operation, the employed FT-IR was equipped with a custom-built high pressure demountable cell (Harrick) that was optimized for the best possible signal-to-noise ratio. ${ }^{35}$ The ZnSe cell had a thickness $150 \mu \mathrm{m}^{35}$ and allowed for a maximum working pressure of $25 \mathrm{MPa}$. The data generated by the FTIR were digitally read out by a specific software (Spectra Manager by Jasco) and the variation of the solute concentration over time was monitored through the absorbance spectra at wavenumbers corresponding to different vibration modes. The procedure to select the working wavenumbers, the experimental protocol and the fitting procedure have been reported in ref. 65 and 66.

\section{Results}

\subsection{Critical line and Widom line}

The $\mathrm{CO}_{2}$ + ethanol mixture was investigated by EMD simulation along the isobar $p=10 \mathrm{MPa}$ in the temperature range between $T=305$ and $340 \mathrm{~K}$ with a composition $x_{\mathrm{CO} 2}=0.97 \mathrm{~mol} \mathrm{~mol}^{-1}, c f$. Fig. 2. This composition was chosen as the closest to the infinite dilution limit that allowed for adequate statistics. Along this path, the mixture undergoes two transitions, indicated as I and II in Fig. 2. First, the Widom line is crossed at $T \sim 323 \mathrm{~K}$ (point I) marking the transition from liquid-like to gas-like conditions, as indicated by the maxima of the response functions $c_{\mathrm{p}}, \alpha_{\mathrm{v}}, \beta_{\mathrm{T}}, c f$. Fig. S2-S4 in the ESI. $\dagger$ Each function's inflection point corresponds to a maximum of a thermodynamic response function and consequently determines the mixture's Widom line. The inflection points of the enthalpy and density, which correspond to maxima of the isobaric heat capacity $c_{\mathrm{p}}$ and the thermal expansion $\alpha_{\mathrm{v}}$, respectively, occur according to the employed force field at $T=317 \mathrm{~K}$ for pure $\mathrm{CO}_{2}$ and $T=323 \mathrm{~K}$ for the mixture, $c f$. Fig. 3. Thus, the addition of a small amount of ethanol shifts the Widom line up by $\sim 6 \mathrm{~K}$. To assess the capability of the employed force field to predict the studied properties, the temperature dependence of density and enthalpy of pure $\mathrm{CO}_{2}$ was compared to the Span-Wagner equation of state, ${ }^{67}$ which is of reference quality. In general, the employed $\mathrm{CO}_{2}$ force field is able to predict the density of $\mathrm{CO}_{2}$ in the studied temperature and pressure ranges with a good accuracy. The average deviation between simulation results and the Span-Wagner equation of state ${ }^{67}$ is $5.4 \%$.

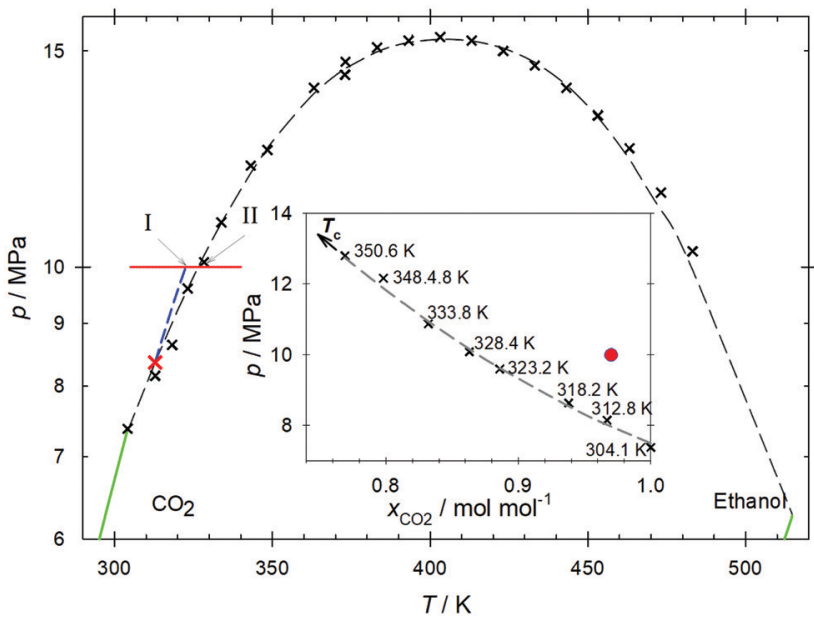

Fig. 2 Pressure-temperature projection of both components' vapor pressure curves (green lines). The critical line of the mixture (dashed line) was determined on the basis of experimental literature data ${ }^{68,69}$ (black crosses). The Widom line (dashed blue line) extends the influence of the present mixture's ( $x_{\mathrm{CO} 2}=0.97 \mathrm{~mol} \mathrm{~mol}^{-1}$ ) critical point (red cross) to higher temperatures and pressures. The red line represents the studied isobar in the homogeneous region. Points I and II represent the crossing of the Widom line and the transition from the super- to the subcritical regimes. The inset shows the critical line (dashed line) as a function of mole fraction as given in Table 2 (black crosses). The red bullet symbolizes the molar composition $x_{\mathrm{CO} 2}=0.97 \mathrm{~mol} \mathrm{~mol}^{-1}$ and pressure $p=10 \mathrm{MPa}$ at which all molecular dynamics simulations were carried out. 


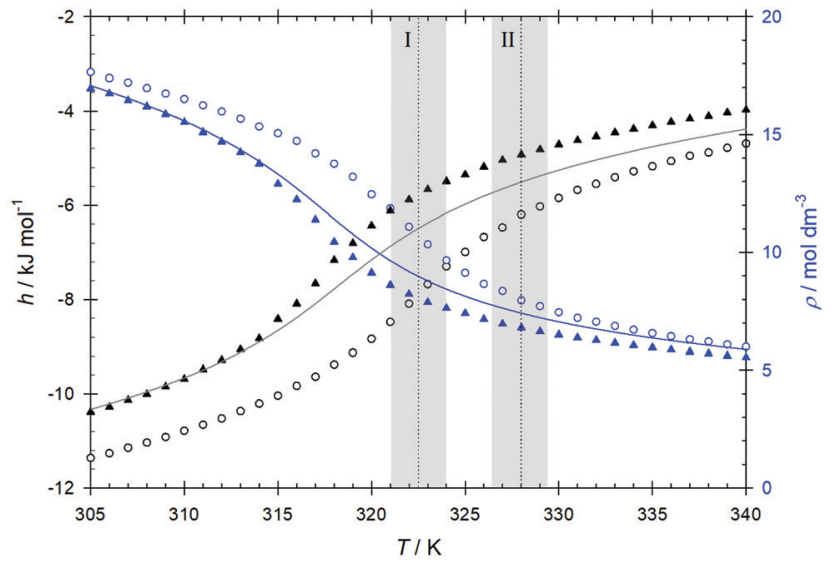

Fig. 3 EMD simulation results for enthalpy $h$ (black) and density $\rho$ (blue) of pure $\mathrm{CO}_{2}$ (triangles) and the $\mathrm{CO}_{2}$-ethanol mixture (circles) to determine the Widom line at pressure $p=10 \mathrm{MPa}$. The lines represent the properties of pure $\mathrm{CO}_{2}$ calculated with the Span-Wagner equation of state. ${ }^{67}$ Statistical uncertainties are within symbol size. The temperatures I and II represent the crossing of the Widom line and the transition from the super- to the subcritical regimes. The shaded areas indicate their expected uncertainty.

However, the predicted inflection points, which define the Widom line, occur approximately $2 \mathrm{~K}$ below those according to the Span-Wagner equation of state. ${ }^{67}$ Therefore, the related uncertainty of the present simulation results is expected to have a similar magnitude, which is marked as a shaded region around the calculated transition points I and II.

After crossing the Widom line, the mixture undergoes a transition from the supercritical gas-like domain to a gas at subcritical conditions at $T \sim 328 \mathrm{~K}$ (point II), as indicated by the critical line of the mixture, $c f$. Table 2 . More specifically, while for $T=318 \mathrm{~K}$ all states above $p=8.64 \mathrm{MPa}$ and $x_{\mathrm{CO} 2}=0.938 \mathrm{~mol} \mathrm{~mol}^{-1}$ are located in the supercritical region, for $T=328 \mathrm{~K}$ only states above $p=10.09 \mathrm{MPa}$ and $x_{\mathrm{CO} 2}=0.863 \mathrm{~mol} \mathrm{~mol}^{-1}$ lie in the supercritical region. This can be clearly seen in the inset of Fig. 2, while for temperatures below $T \sim 328 \mathrm{~K}$ the simulated pressure-composition pair is clearly located above the critical line, for higher temperatures, the simulated pressure is below that of the critical line. The pressure-temperature projection of the vapor pressure curves for the $\mathrm{CO}_{2}+$ ethanol mixture is shown in Fig. 2. The critical line of the mixture was determined by joining the mixture's critical points from experimental data. ${ }^{67-70}$ A distinctive feature of the curve is that it goes through a pronounced pressure maximum at $p \sim 15.5 \mathrm{MPa}$ despite the

Table 2 Selected critical points of the studied mixture from experimental data $^{67-70}$

\begin{tabular}{lcl}
\hline$T / \mathrm{K}$ & $p / \mathrm{MPa}$ & $x_{\mathrm{CO}_{2}} / \mathrm{mol} \mathrm{mol}^{-1}$ \\
\hline 304.13 & 7.38 & 1.0 \\
312.82 & 8.15 & 0.967 \\
318.24 & 8.64 & 0.938 \\
328.36 & 10.09 & 0.863 \\
333.82 & 10.88 & 0.832 \\
350.62 & 12.80 & 0.769 \\
514.71 & 6.27 & 0.0
\end{tabular}

relatively small difference between the critical pressures of pure $\mathrm{CO}_{2}$ and pure ethanol, $p_{\mathrm{c}}=7.4$ and 6.3 MPa, respectively. The large temperature range of that curve ( $304 \mathrm{~K}$ to $515 \mathrm{~K}$ ) is simply due to the difference between the critical temperatures of the pure substances. Note that the composition varies along the critical line such that the pressure-composition pair that is focussed on does not cross the two-phase region.

\subsection{Intradiffusion coefficients}

The dynamical crossover between the supercritical high density and the low density regimes across the Widom line has been related to the presence of inflection points or extrema of some transport coefficients. ${ }^{3}$ Therefore, the analysis of the diffusion behavior of the mixture along the regarded isobar offers insight into the underlying transition dynamics. The temperature dependence of the intradiffusion coefficient of $\mathrm{CO}_{2}$ is sigmoidal, with an initially rather weak temperature dependence followed by a strong stepwise increase at temperatures between $T=320$ and $330 \mathrm{~K}, c f$. Fig. 4 . This curve is similar to that observed for the enthalpy and shows an inflection point at the Widom line, i.e. at $T \sim 323 \mathrm{~K}$. The random propagation of molecules is not only dependent on the thermodynamic state point, but is also strongly affected by other factors like molecular size and polarity. Thus, within a mixture, the components generally propagate with different velocity distributions leading to different intradiffusion coefficients. In fact, the intradiffusion coefficient of the bulkier ethanol molecules is on average $40 \%$ lower than that of the smaller $\mathrm{CO}_{2}$ molecules. Although both curves show a sigmoidal behavior, the stepwise increase of the intradiffusion coefficient of ethanol is less pronounced than the one of $\mathrm{CO}_{2}$, which can be linked to the presence of local density inhomogeneities caused by self-association. Further, the inflection point

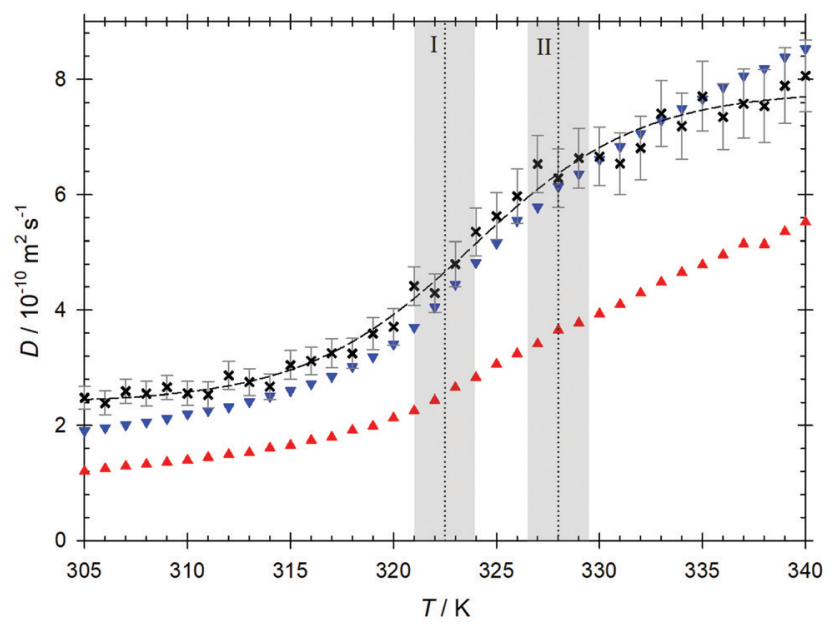

Fig. 4 EMD simulation results for the intradiffusion coefficients of ethanol (red triangles) and $\mathrm{CO}_{2}$ (blue triangles) as well as the Maxwell-Stefan diffusion coefficient (black symbols) along the isobar $p=10 \mathrm{MPa}$. The statistical uncertainties of the intradiffusion coefficients are within symbol size. The dashed line serves as a guide to the eye. The temperatures I and II represent the crossing of the Widom line and the transition from the super- to the subcritical regimes. The shaded areas indicate their expected uncertainty. 
of the curve is observed at the transition between the super- and subcritical regimes $T \sim 328 \mathrm{~K}$.

\subsection{Microscopic structure}

To elucidate the relevant microscopic structure aspects, the center-of-mass radial distribution functions of the ethanolethanol $g_{\text {EtOH-EtOH }}(r), \mathrm{CO}_{2}$-ethanol $g_{\mathrm{CO} 2-\mathrm{EtOH}}(r)$ and $\mathrm{CO}_{2}-\mathrm{CO}_{2}$ $g_{\mathrm{CO} 2-\mathrm{CO} 2}(r)$ pair interactions were analyzed, $c f$. Fig. 5 and Fig. S5 of the ESI. $\dagger$ The main peak of $g_{\text {EtOH-EtOH }}(r)$ with a maximum located at $r \sim 4.3 \AA$ is related to ethanol self-association through hydrogen bonding. This first peak is followed by a well defined shoulder, which is more pronounced at the lowest studied temperature and indicates an overlap with the second solvation shell. The location of the main peak does not change significantly along the studied isobar, suggesting that ethanol remains structured at short intermolecular distances. On the other hand, $g_{\mathrm{CO} 2-\mathrm{CO} 2}(r)$ shows a well defined first peak followed by a considerably smaller second peak, which becomes weaker with increasing temperature and disappears at $T \sim 330 \mathrm{~K}$. This reduction of the long-range structure is expected because of the transition from supercritical gas-like to compressed gas conditions. The observed differences in peak intensity and the relatively small main peaks observed in the radial distribution functions at lower temperatures are mainly a result from

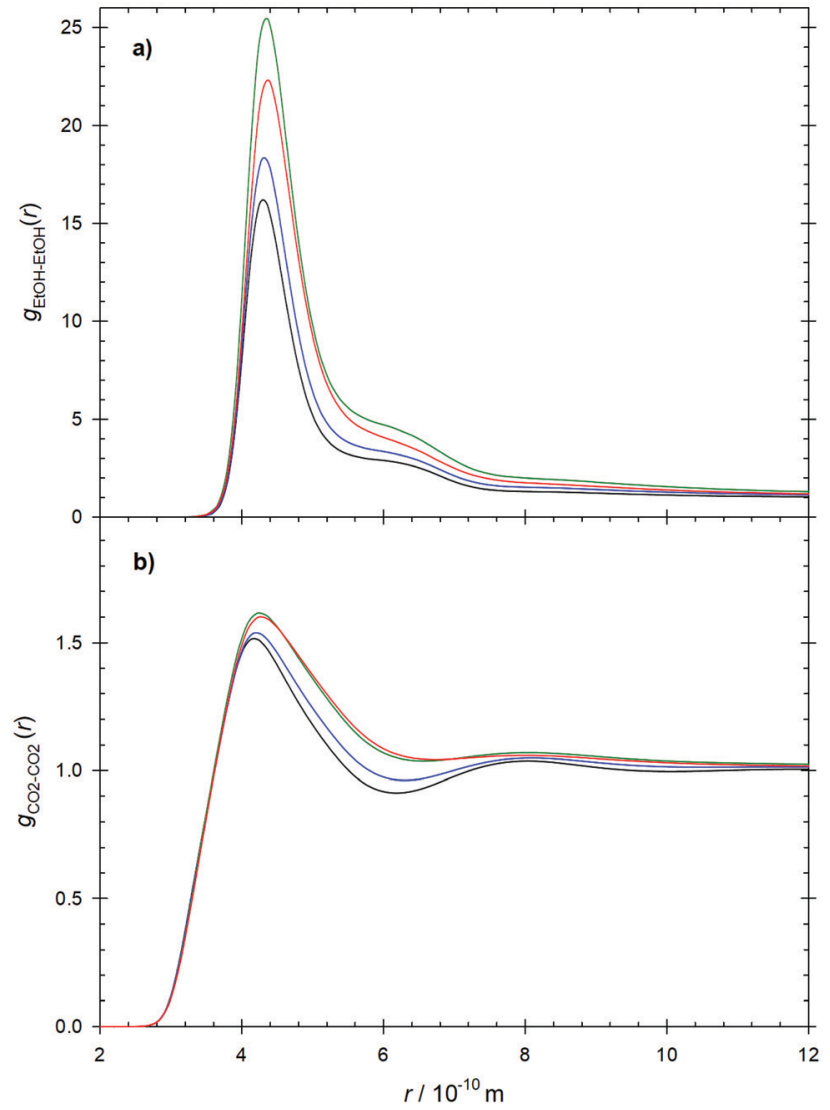

Fig. 5 (a) Ethanol-ethanol and (b) $\mathrm{CO}_{2}-\mathrm{CO}_{2}$ radial distribution functions at $T=310 \mathrm{~K}$ (black), $320 \mathrm{~K}$ (blue), $330 \mathrm{~K}$ (green) and $340 \mathrm{~K}$ (red) along the isobar $p=10 \mathrm{MPa}$. statistical standardization, i.e. more ethanol or $\mathrm{CO}_{2}$ molecules can be found in the far range of the simulation volume. ${ }^{71}$ The $\mathrm{CO}_{2}$-ethanol pair interaction, shown in Fig. S5 in the ESI, $\dagger$ exhibits a relatively small peak with a shoulder located between $r \sim 3.0$ and $6.3 \AA$, which partially lies within the range of hydrogen bonding interactions and suggests the occurrence of relatively strong $\mathrm{CO}_{2}$-ethanol association. ${ }^{32}$ However, because of the rather small peak magnitude, these interactions are expected to occur rather sporadically.

A quantification of the local inhomogeneities can be achieved by monitoring the average coordination numbers for the first coordination shell defined as $N_{x-y}=4 \pi \rho_{y} \int_{0}^{r_{\mathrm{c}}} r^{2} g_{x-y}(r) \mathrm{d} r$. Therein, $x$ stands for the central interaction site surrounded by interaction sites of type $y, \rho_{y}$ is the bulk density of interaction sites of type $y, g_{x-y}(r)$ represents the radial distribution function of the pair involved in the running average number calculation and $r_{\mathrm{c}}$ is the radius of the first coordination shell, i.e. the location of the first minimum of the regarded radial distribution function $g_{x-y}(r)$. The average coordination number $N_{\mathrm{CO} 2-\mathrm{CO} 2}$ shows a similar behavior as the bulk density. At temperatures between $T=315$ and $330 \mathrm{~K}$, the coordination number decreases rapidly to approximately one third of its initial value and remains more stable in the compressed gas region, $c f$. Fig. 6. The strong decrease of the coordination number is mirrored in the observed stepwise increase of the $\mathrm{CO}_{2}$ intradiffusion coefficient. Similarly, the inflection point of the coordination number was also found to be located at the Widom line, i.e. at $T \sim 323 \mathrm{~K}$. The average coordination number $N_{\mathrm{EtOH}-\mathrm{EtOH}}$, indicating the amount of ethanol self-association, shows a stronger temperature dependence in the supercritical liquid-like region. At $T=305 \mathrm{~K}$, each ethanol molecule is associated on average with 1.4 alcohol molecules, but after an increase of $10 \mathrm{~K}$ in temperature this value is reduced to 1.23 . This implies a reduction of about $12 \%$ in the hydrogen bonded structures and explains the related increase of the ethanol intradiffusion coefficient between

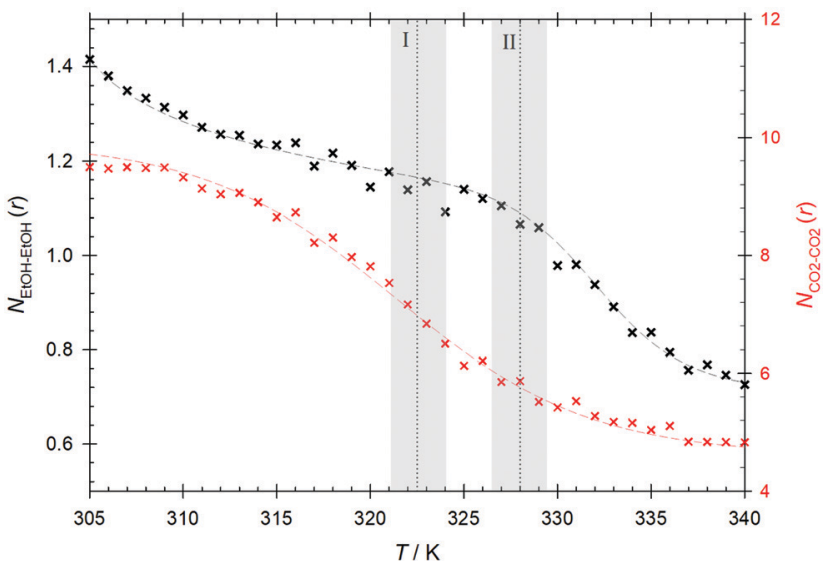

Fig. 6 Average coordination number of the ethanol-ethanol and $\mathrm{CO}_{2}-$ $\mathrm{CO}_{2}$ pairs as a function of temperature along the isobar $p=10 \mathrm{MPa}$. The dashed lines serve as a guide to the eye. The temperatures I and II represent the crossing of the Widom line and the transition from the super- to the subcritical regimes. The shaded areas indicate their expected uncertainty. 
$T=305$ and $315 \mathrm{~K}$. In the region between 315 and $328 \mathrm{~K}$, the decrease of the average ethanol-ethanol coordination number is much less pronounced than that of $\mathrm{CO}_{2}$, suggesting the presence of enhanced ethanol hydrogen bonded structures that are disrupted to a lesser extent by the strong density reduction. These rather stable ethanol hydrogen bonded structures are linked to mixture microheterogeneities observed in the domain influenced by the Widom line and the super- to subcritical transition. The relatively small values of the thermodynamic factor as well as the milder sigmoidal increase of the ethanol intradiffusion coefficient can also be explained with these microscopic structures. At temperatures above $T \sim 328 \mathrm{~K}$, the coordination number decreases almost stepwise, suggesting a steady breakup of the hydrogen bonded structures with temperature in the compressed gas region.

The presence of ethanol self-association and microheterogeneities can be visually corroborated when simulation snapshots are analyzed. Although a snapshot represents only one microstate of a molecular system, in case of mixtures with associating components, a single microstate may well represent all possible microstates, since they are permutations of the segregation patterns. ${ }^{72}$ Fig. 7 shows snapshots of simulation volumes for four temperatures. In spite of the low alcohol content, ethanol molecules tend to self-associate and form hydrogen bonded networks. At low temperatures up to the Widom line, most of the ethanol molecules are part of clusters that form segregated domains. As the temperature is increased, the ethanol clusters become smaller and are more uniformly distributed in the simulation volume. This observation corresponds to the observed values of the intradiffusion coefficient of ethanol and the relatively low values of the thermodynamic factor.

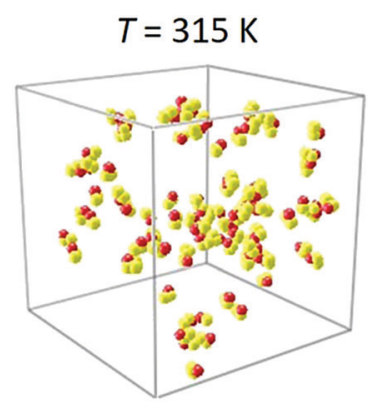

$T=328 \mathrm{~K}$
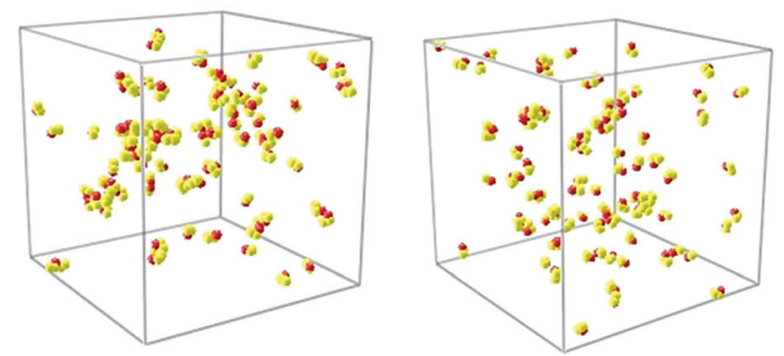

Fig. 7 Snapshots of the present EMD simulations at selected temperatures The $\mathrm{CO}_{2}$ solvent molecules were graphically removed to reveal clustering among ethanol molecules.

\subsection{Mutual diffusion coefficients}

The Maxwell-Stefan diffusion coefficient calculated from the Onsager phenomenological coefficients ${ }^{73}$ of the studied mixture is shown in Fig. 4. As can be seen for temperatures below $T=328 \mathrm{~K}$, the Maxwell-Stefan diffusivity curve runs almost parallel to and above the one of $\mathrm{CO}_{2}$ intradiffusion. Higher values of the Maxwell-Stefan diffusion coefficient with respect to the intradiffusion coefficient are related to cluster formation due to alcohol self-association. ${ }^{74}$ In the regime following the transition to the compressed gas, the MaxwellStefan diffusion coefficient becomes lower than the $\mathrm{CO}_{2}$ intradiffusion coefficient, which is in line with the strong reduction of the ethanol hydrogen bonded structures indicated by the decrease of the average ethanol-ethanol coordination number. The inflection point of the Maxwell-Stefan diffusivity curve is located in the vicinity of the Widom line, i.e. at $T \sim 324 \mathrm{~K}$.

The Maxwell-Stefan diffusion coefficient obtained from EMD simulation can be straightforwardly related to the Fick diffusion coefficient $D$ through the so-called thermodynamic factor $\Gamma$, i.e. $D=\Gamma Ð$, which is a measure of the mixture's nonideality. Because of the nature of the $\mathrm{CO}_{2}+$ ethanol mixture and the presence of microheterogeneities, the sampled thermodynamic factor reaches relatively low values $\Gamma \sim 0.45$. The expected minimum of the thermodynamic factor in the proximity of the Widom line is predicted clearly by the employed equations of state. The Kirkwood-Buff integration results predict a weaker minimum shifted to higher temperatures, $c f$. Fig. 8. For the sake of consistency and because of the rather large differences in $\Gamma$ obtained from the different equations of state, the thermodynamic factor from Kirkwood-Buff integration was employed here to calculate the Fick diffusion coefficient. Since the values of the sampled thermodynamic factor do

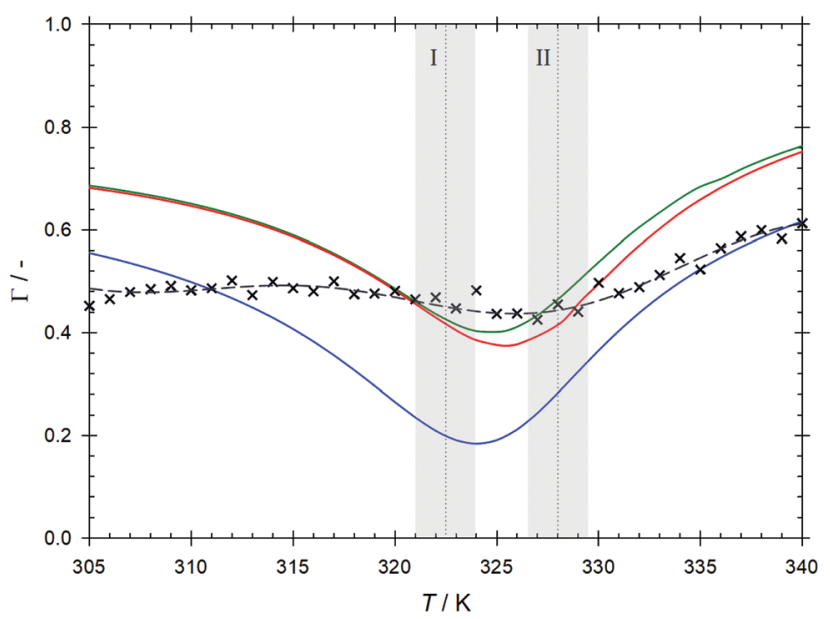

Fig. 8 Thermodynamic factor $\Gamma$ of the $\mathrm{CO}_{2}+$ ethanol mixture as a function of temperature along the isobar $p=10 \mathrm{MPa}$. The solid lines represent results from equations of state, i.e. Peng-Robinson (green), Soave-Redlich-Kwong (red) and PCP-SAFT (blue). The symbols indicate simulation results from Kirkwood-Buff integration. The dashed line serves as a guide to the eye. The temperatures I and II represent the crossing of the Widom line and the transition from the super- to the subcritical regimes. The shaded areas indicate their expected uncertainty. 
not show a strong variation in the range of the studied thermodynamic conditions, the temperature dependence of the sampled Fick diffusion coefficient is similar to that observed for the Maxwell-Stefan diffusion coefficient, $c f$. Fig. 9. However, its inflection point is shifted to a higher temperature, i.e. $T \sim 329 \mathrm{~K}$, which can be explained with the changes observed for the ethanol-ethanol average coordination number near this temperature. In general, values of the Fick diffusion coefficient sampled by EMD are comparable with present measurements within the statistical uncertainties, $c f$. Table 3. However, the experimental values exhibit a smoother temperature dependence with an inflection point located at $T \sim 327 \mathrm{~K}$. The comparably lower values of the predicted Fick diffusion coefficient in the supercritical liquid-like region, i.e. at $T<320 \mathrm{~K}$, could be due to an overestimation of ethanol selfassociation by molecular simulation. Approaching the Widom line, where density variations are large, simulation and experimental results agree quite well up to $T \sim 332 \mathrm{~K}$, i.e. right after the transition into the subcritical region. At the highest studied

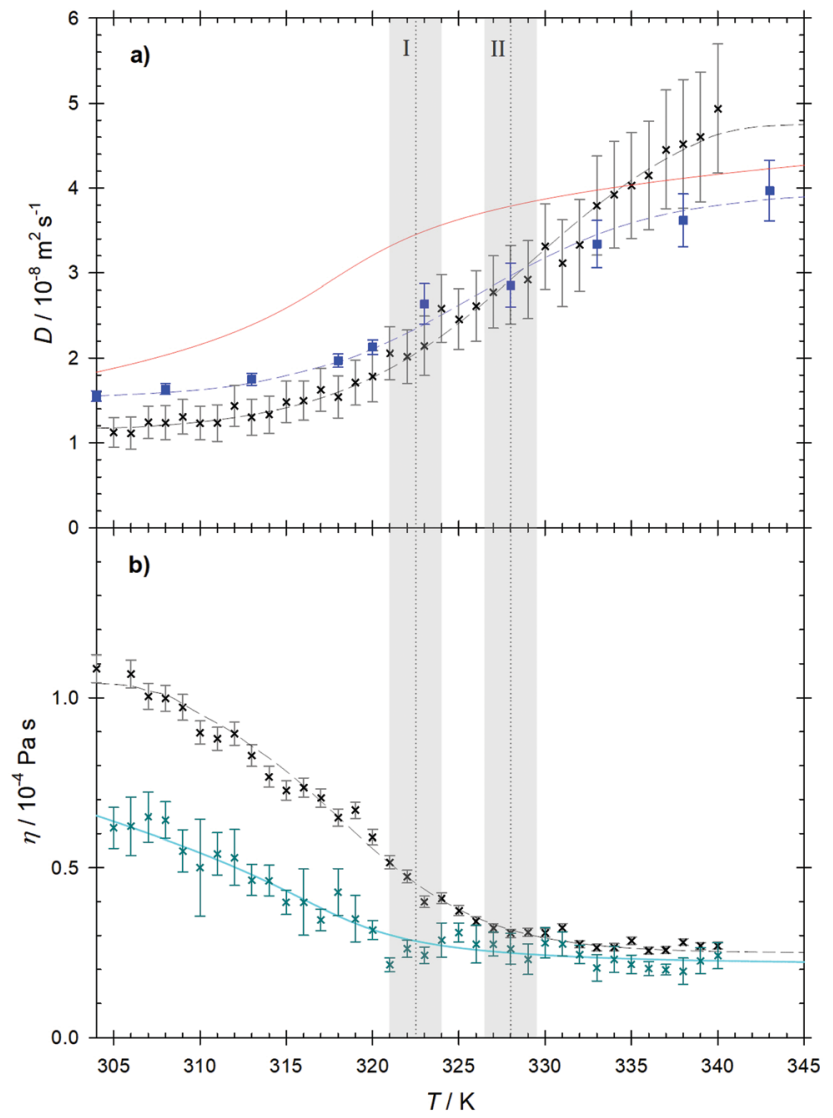

Fig. 9 (a) Fick diffusion coefficient predicted by EMD simulation (crosses) and obtained experimentally (squares) as a function of temperature along the isobar $p=10 \mathrm{MPa}$. The red line represents the predictive equation by $\mathrm{He}$ and $\mathrm{Yu}^{82}$ (b) Shear viscosity predicted by EMD simulation (crosses) compared to the shear viscosity of pure $\mathrm{CO}_{2}$ according to the correlation by Laesecke and Muzny ${ }^{84}$ (cyan line). The dashed lines serve as a guide to the eye. The temperatures I and II represent the crossing of the Widom line and the transition from the super- to subcritical regimes. The shaded areas indicate their expected uncertainty. temperatures, the Fick diffusion coefficient predicted by EMD is overestimated when compared with the experimental data. This suggests that the actual breakup of ethanol hydrogen bonded structures in the compressed gas region occurs more gradually than predicted by EMD simulation. It should be noted that contrary to EMD simulations, where the mixture concentration is exactly known, the experimental concentration can only be estimated. Therefore, the differences between simulation and experimental values can partially be explained with the difference in ethanol concentration, which has been proven to have a strong influence on the Fick diffusion coefficient even in the dilution limit of $\mathrm{scCO}_{2}$ mixtures. ${ }^{66,75}$ This significant influence of concentration on the Fick diffusion coefficient is mainly related to the strong concentration dependence of the thermodynamic factor given by the proximity of the critical point. ${ }^{66}$ A direct comparison of present experimental results with data from previous studies ${ }^{36,37}$ is not possible because of they were measured for different state points.

The Stokes-Einstein based equations by Wilke-Chang, ${ }^{76}$ Sassiat, ${ }^{77}$ Tyn-Calus, ${ }^{78}$ Scheibel, $^{76}$ Reddy-Doraiswamy ${ }^{79}$ and Lai-Tan ${ }^{80}$ as well as the free volume based equations by Catchpole and King, ${ }^{81} \mathrm{He}$ and $\mathrm{Yu}^{82}$ as well as Funazukuri et al. ${ }^{83}$ were tested with respect to their ability to predict the Fick diffusion coefficient at infinite dilution of the $\mathrm{CO}_{2}+$ ethanol mixture. However, none of these equations was able to capture neither qualitatively nor quantitatively the present experimental Fick diffusion coefficient values, $c f$. Fig. S7 of the ESI. $\dagger$ The predictive $\mathrm{He}$ and $\mathrm{Yu}^{82}$ equation yields the best results among the tested equations, however, its agreement with the present experimental results is quite poor in the supercritical region, $c f$. Fig. 9(a). The strong overestimation of the Fick diffusion coefficient in regions with a higher density, where ethanol selfassociation plays a decisive role for molecular mobility, as well as the better agreement with experiments in the compressed gas region, indicate a failure of these models to adequately consider the hydrogen bonded structures in the mixture.

\subsection{Shear viscosity}

The importance of considering information on hydrogen bonding dynamics for the prediction of transport properties can be clearly

Table 3 Experimental Fick diffusion coefficient of the $\mathrm{CO}_{2}+$ ethanol mixture along the isobar $p=10 \pm 0.05 \mathrm{MPa}$. The listed values represent the average $D$ and standard deviation $\sigma$ of typically ten different measurements. The given temperatures have an uncertainty of $\pm 0.1 \mathrm{~K}$

\begin{tabular}{lll}
\hline$T / \mathrm{K}$ & $D / 10^{-8} \mathrm{~m}^{2} \mathrm{~s}^{-1}$ & $\sigma / 10^{-8} \mathrm{~m}^{2} \mathrm{~s}^{-1}$ \\
\hline 304 & 1.55 & 0.06 \\
308 & 1.64 & 0.07 \\
313 & 1.75 & 0.07 \\
318 & 1.97 & 0.08 \\
320 & 2.07 & 0.09 \\
323 & 2.43 & 0.23 \\
328 & 2.86 & 0.25 \\
333 & 3.34 & 0.28 \\
338 & 3.62 & 0.31 \\
343 & 3.97 & 0.36
\end{tabular}


observed when the shear viscosity is analyzed. Fig. 9(b) shows the strong difference between the shear viscosity of pure $\mathrm{CO}_{2}$ and that of the mixture. In the liquid-like region, where intermolecular forces dominate the viscous effects, the shear viscosity of the mixture exhibits an increment of approximately $70 \%$ from the value of pure $\mathrm{CO}_{2}$. This enhancement can be ascribed to the presence of microheterogeneities caused by hydrogen bonding networks despite the small amount of ethanol present in the mixture. Here, ethanol self-association is expected to play the main role in the formation of microscopic structures because of the low values of the $\mathrm{CO}_{2}$-ethanol coordination number, i.e. $N_{\mathrm{CO}_{2} \text {-EtOH }}<0.3$. As higher temperatures are reached, the momentum transfer due to molecular thermal motion makes an increasingly important contribution to the shear viscosity and hydrogen bonds break, reducing the viscosity enhancement to less than $15 \%$ in the compressed gas region. Further, the shear viscosity curve of the mixture shows an inflection point located at $T \sim$ $320 \mathrm{~K}$, which cannot be observed for pure $\mathrm{CO}_{2}$. Viscosity and diffusion curves exhibit an opposing behavior, as expected from the Stokes-Einstein equation.

\section{Conclusions}

A study on the dynamic behavior of ethanol diluted in $-\mathrm{CO}_{2}$ in the temperature range from $T=304$ to $343 \mathrm{~K}$ along the isobar $p=10 \mathrm{MPa}$ was conducted employing complementary approaches, i.e. experiment and molecular simulation. Along this path, the studied mixture goes through a transition in the supercritical region by crossing the Widom line and from super- to subcritical regimes. The Fick diffusion coefficient was measured with the Taylor dispersion technique, while several EMD simulation methods were employed. Shear viscosity, intra- and Maxwell-Stefan diffusion coefficients were sampled with equilibrium molecular dynamics, employing rigid, non-polarizable force fields based on Lennard-Jones sites and superimposed point charges or quadrupoles, and the Green-Kubo formalism. The thermodynamic factor was calculated with Kirkwood-Buff integration. In this way, the Fick diffusion coefficient was determined by EMD simulations consistently on the basis of the selected force fields. In order to validate the force fields employed to describe the mixture, the VLE of the mixture was calculated at three temperatures and compared successfully with experimental data from the literature. Further, to gain insight into the microscopic structure of the mixture, center-of-mass radial distribution functions as well as averaged coordination numbers were investigated.

In the region where the crossover between the liquid-like and gas-like regimes occurs, several thermophysical properties become very sensitive to temperature and pressure variations and can therefore change drastically along supercritical paths. Along the studied isobar, the presence of inflection points in the temperature dependence of density, enthalpy, shear viscosity, intra- and transport diffusion coefficients were reported. Mostly, the inflection points were observed at temperatures between $T \sim 321$ and $\sim 324 \mathrm{~K}$, a range that specifies the region influenced by the Widom line. Moreover, the calculated values of the center-of-mass average coordination numbers up to the first solvation shell also exhibit an inflection point in this region, shedding light on the underlying microscopic structural background of the observed macroscopic property behavior.

The transition between the supercritical gas-like and the subcritical compressed gas regimes at $T \sim 328 \mathrm{~K}$ is smooth for most of the studied properties, i.e. density, enthalpy, shear viscosity, Maxwell-Stefan and $\mathrm{CO}_{2}$ intradiffusion coefficients. However, the intradiffusion coefficient of ethanol and the Fick diffusion coefficient showed an inflection point in the proximity of this transition. It was noticed that the averaged coordination number of ethanol-ethanol pairs, which is an indicator of ethanol self-association, shows an almost stepwise change in this region. This was related to a relatively strong decrease of ethanol self-association when moving from supercritical to subcritical states, which could also be observed by analyzing snapshots of the simulation volumes.

A satisfactory agreement was found between predictive EMD simulation data and experimental results for the Fick diffusion coefficient, especially in the temperature range from the Widom line to the super- to subcritical transition. Both experiment and simulation exhibit a sigmoidal behavior along the studied isobar, however, experiments showed an inflection point at a slightly lower temperature. Possible reasons for the difference between simulation and experimental results were thoroughly discussed.

An analysis of the shear viscosity of the mixture corroborated the strong influence of microheterogeneities given by hydrogen bonded networks on the macroscopic transport properties of the mixture.

\section{Conflicts of interest}

There are no conflicts to declare.

\section{Acknowledgements}

All authors greatly acknowledge the support of Prof. Joachim Gross (University of Stuttgart) regarding the handling of the PCP-SAFT equation of state. The authors Y. G. and V. S. kindly appreciate financial support by the PRODEX program of the Belgian Federal Science Policy Office and the European Space Agency (ESA). The authors R. C., G. G.-C. and J. V. want to acknowledge the support by - Deutsche Forschungsgemeinschaft (DFG) under Grant No. VR $6 / 11$. This work was carried out under the auspices of the Boltzmann-Zuse Society (BZS). Equilibrium molecular dynamics simulations were performed either on Cray's CS500 system Noctua at the Paderborn Center for Parallel Computing $\left(\mathrm{PC}^{2}\right)$ or on the HPE Apollo system Hawk at the High Performance Computing Centre Stuttgart (HLRS) contributing to the project MMHBF2.

\section{Notes and references}

1 M. Mukhopadhyay, Natural extraction using supercritical carbon dioxide, CRC Press, 1st edn, 2000. 
2 R. Gupta and J.-J. Shim, Solubility in supercritical carbon dioxide, CRC Press, 1st edn, 2007.

3 F. A. Gorelli, T. Bryk, M. Kirsch, G. Ruocco, M. Santoro and T. Scopigno, Sci. Rep., 2013, 3, 1203.

4 J. Losey and R. J. Sadus, J. Phys. Chem. B, 2019, 123, 8268-8273.

5 K. Karalis, C. Ludwig and B. Niceno, Sci. Rep., 2019, 9, 15731.

6 Y. D. Fomin, V. N. Ryzhov, E. N. Tsiok and V. V. Brazhkin, Phys. Rev. E: Stat., Nonlinear, Soft Matter Phys., 2015, 91, 022111.

7 A. Imre, G. Hazi, A. Horvath, C. Maraczy, V. Mazur and S. Artemenko, Nucl. Eng. Des., 2011, 241, 296-300.

8 A. Imre, C. Ramboz, U. Deiters and K. Kraska, Environ. Earth Sci., 2015, 73, 4373-4384.

9 M. Raju, D. T. Banuti, P. C. Ma and M. Ihme, Sci. Rep., 2017, 7, 3027.

10 N. Polikhronidi, R. Batyrova, A. Aliev and I. Abdulagatov, J. Therm. Sci., 2019, 28, 394-430.

11 P. Raveendran, Y. Ikushima and S. L. Wallen, Acc. Chem. Res., 2005, 38, 478-485.

12 T. Tassaing, P. Lalanne, S. Rey, F. Cansell and M. Besnard, Ind. Eng. Chem. Res., 2000, 39, 4470-4475.

13 I. Skarmoutsos and E. Guardia, J. Phys. Chem. B, 2009, 111, 8887-8897.

14 K. Nishikawa and I. Tanaka, Chem. Phys. Lett., 1995, 244, 149-152.

15 H. Nakayama, K.-I. Saitow, M. Sakashita, K. Ishii and K. Nishikawa, Chem. Phys. Lett., 2000, 320, 323-327.

16 N. Nishikawa and T. Morita, Chem. Phys. Lett., 2000, 316, 238-242.

17 G. G. Simeoni, T. Bryk, F. A. Gorelli, M. Kirsch, G. Ruocco, M. Santoro and T. Scopigno, Nat. Phys., 2010, 6, 503-507.

18 S. Hans and C. C. Yu, Phys. Rev. E: Stat., Nonlinear, Soft Matter Phys., 2012, 85, 051201.

19 P. Gallo, D. Corradini and M. Rovere, Nat. Commun., 2014, 5, 5806.

20 M. Y. Ha, T. J. Yoon, T. Tlusty, Y. Jho and W. B. Lee, J. Phys. Chem. Lett., 2018, 9, 1734-1738.

21 M. Fisher and B. Widom, J. Chem. Phys., 1969, 50, 3756-3772.

22 V. Brazhkin, Y. Fomin, A. Lyapin, V. Ryzhov and K. Trachenko, Phys. Rev. E: Stat., Nonlinear, Soft Matter Phys., 2012, 85, 031203.

23 D. Bolmativ, M. Zhernenkov, D. Zav'yalov, S. Tkachev, A. Cunsolo and Y. Cai, Sci. Rep., 2015, 5, 15850.

24 L. Xu, P. Kumar, S. Buldyrev, S.-H. Chen, P. Poole, F. Sciortino and H. Stanley, Proc. Natl. Acad. Sci. U. S. A., 2005, 102, 16558-16562.

25 D. Banuti, M. Raju and M. Ihme, J. Supercrit. Fluids, 2020, 165, 104895.

26 T. J. Yoon, M. Y. Ha, W. B. Lee and Y.-W. Lee, J. Chem. Phys., 2018, 149, 014502.

27 T. J. Yoon, M. Y. Ha, W. B. Lee and Y.-W. Lee, J. Chem. Phys., 2019, 150, 154503.

28 E. Mareev, V. Aleshkevich, F. Potemkin, V. Bagratashvili, N. Minaev and V. Gordienko, Opt. Express, 2018, 26, 13229-13238.
29 B. Chu, Ber. Bunsen-Ges., 1972, 76, 202-215.

30 M. M. Hoffmann and M. S. Conradi, J. Phys. Chem. B, 1998, 102, 263-271.

31 M. Saharay and S. Balasubramanian, J. Phys. Chem. B, 2006, 110, 3782-3790.

32 W. Xu, J. Yang and Y. Hu, J. Phys. Chem. B, 2009, 113, 4781-4789.

33 T. Aida, T. Aizawa, M. Kanakubo and H. Nanjo, J. Phys. Chem. B, 2010, 114, 13628-13636.

34 B. A. McGuire, M.-A. Martin-Dumel and M. C. McCarthy, J. Phys. Chem. A, 2017, 121, 6283-6287.

35 Y. Gaponenko, V. Gousselnikov, C. I. A. V. Santos and V. Shevtsova, Microgravity Sci. Technol., 2019, 31, 475-486.

36 D. Mei, H. Li and W. Wang, Huagong Xuebao, 1995, 46, 357-364.

37 C. Y. Kong, T. Funazukuri and S. Kagei, J. Supercrit. Fluids, 2006, 37, 359-366.

38 Y. Houndonougbo, K. Kuczera, B. Subramaniam and B. B. Laird, Mol. Simul., 2007, 33, 861-869.

39 L. Hui, W. Rui, F. Weiyu, L. Zhaomin, M. Tao and N. Guozhi, Acta Pet. Sin., 2015, 31, 78-83.

40 I. Skarmoutsos, E. Guardia and J. Samios, J. Chem. Phys., 2010, 133, 014504.

41 W. Xu and J. Yang, J. Phys. Chem. A, 2010, 114, 5414-5428.

42 S. Reiser, N. McCann, M. Horsch and H. Hasse, J. Supercrit. Fluids, 2012, 68, 94-103.

43 Z. Li, S. Lai, W. Gao and L. Chen, Russ. J. Phys. Chem. A, 2018, 92, 1332-1337.

44 T. Schnabel, J. Vrabec and H. Hasse, Fluid Phase Equilib., 2005, 223, 134-143.

45 J. Vrabec, J. Stoll and H. Hasse, J. Phys. Chem. B, 2001, 105, 12126-12133.

46 K. Suzuki, H. Sue, M. Itou, R. L. Smith, H. Inomata, K. Arai and S. Saito, J. Chem. Eng. Data, 1990, 35, 63-66.

47 J. S. Lim, Y. Y. Lee and H. S. Chun, J. Supercrit. Fluids, 1994, 7, 219-230.

48 J. W. Ziegler, J. G. Dorsey, T. L. Chester and D. P. Innis, Anal. Chem., 1995, 67, 456-461.

49 L. A. Galicia-Luna, A. Ortega-Rodriguez and D. Richon, J. Chem. Eng. Data, 2000, 45, 265-271.

50 I. Tsivintzelis, D. Missopolinou, K. Kalogiannis and C. Panayiotou, Fluid Phase Equilib., 2004, 224, 89-96.

51 H.-Y. Chiu, M.-J. Lee and H.-M. Lin, J. Chem. Eng. Data, 2008, 53, 2393-2402.

52 C.-N. Han and C.-H. Kang, Korean J. Chem. Eng., 2017, 34, 1781-1785.

53 G. Soave, Chem. Eng. Sci., 1972, 27, 1197-1203.

54 D.-Y. Peng and D. P. Robinson, Ind. Eng. Chem. Fundam., 1976, 15, 59-64.

55 J. Gross and J. Vrabec, AIChE J., 2006, 52, 1194-1204.

56 D. W. Jennings, R. J. Lee and A. S. Teja, J. Chem. Eng. Data, 1991, 36, 303-307.

57 M. S. Green, J. Chem. Phys., 1954, 22, 398-414.

58 R. Kubo, J. Phys. Soc. Jpn., 1957, 12, 570-586.

59 Y. M. Muñoz-Muñoz, G. Guevara-Carrion and J. Vrabec, J. Phys. Chem. B, 2018, 122, 8718-8729. 
60 I.-C. Yeh and G. Hummer, J. Phys. Chem. B, 2004, 108, 15873-15879.

61 S. H. Jamali, L. Wolff, T. M. Becker, A. Bardow, T. J. H. Vlugt and O. A. Moultos, J. Chem. Theory Comput., 2018, 14, 2667-2677.

62 P. Ganguly and N. F. A. van der Vegt, J. Chem. Theory Comput., 2013, 9, 1347-1355.

63 R. Fingerhut and J. Vrabec, Fluid Phase Equilib., 2019, 485, 270-281.

64 G. Rutkai, A. Köster, G. Guevara-Carrion, T. Janzen, M. Schappals, C. W. Glass, M. Bernreuther, A. Wafai, S. Stephan, M. Kohns, S. Reiser, S. Deublein, M. Horsch, H. Hasse and J. Vrabec, Comput. Phys. Commun., 2017, 221, 343-351.

65 S. Ancherbak, C. Santos, J. Legros, A. Mialdun and V. Shevtsova, Eur. Phys. J. E: Soft Matter Biol. Phys., 2016, 39, 111.

66 G. Guevara-Carrion, S. Ancherbak, A. Mialdun, J. Vrabec and V. Shevtsova, Sci. Rep., 2019, 9, 8466.

67 R. Span and W. Wagner, J. Phys. Chem. Ref. Data, 1996, 25, 1509-1596.

68 K. W. Hutcheson and N. R. Foster, Innovations in Supercritical Fluids: Science and Technology, American Chemical Society, 1995.

69 S.-D. Yeo, S.-J. Park, J.-W. Kim and J.-C. Kim, J. Chem. Eng. Data, 2000, 45, 932-935.

70 J. A. Schroeder, S. G. Penoncello and J. S. Schroeder, J. Phys. Chem. Ref. Data, 2014, 43, 043102.
71 C. Oldiges, K. Wittler, T. Tönsing and A. Alijah, J. Phys. Chem. A, 2002, 106, 7147-7154.

72 M. Požar, B. Lovrinčević, L. Zoranić, T. Primorać, F. Sokolić and A. Perera, Phys. Chem. Chem. Phys., 2016, 18, 23971-23979.

73 R. Krishna and J. A. Wesselingh, Chem. Eng. Sci., 1997, 52, 861-911.

74 P. W. M. Rutten, Diffusion in liquids, Delft University Press, 1992.

75 H. Nishiumi and T. Kubota, Fluid Phase Equilib., 2007, 261, 146-151.

76 C. R. Wilke and P. Chang, AIChE J., 1955, 1, 264-270.

77 P. R. Sassiat, P. Mourier, M. H. Caude and R. H. Rosset, Anal. Chem., 1987, 59, 1164-1170.

78 M. T. Tyn and W. F. Calus, J. Chem. Eng. Data, 1975, 20, 106-109.

79 K. A. Reddy and L. K. Doraiswamy, Ind. Eng. Chem. Fundam., 1967, 6, 77-79.

80 C.-C. Lai and C.-S. Tan, Ind. Eng. Chem. Res., 1995, 34, 674-680.

81 O. J. Catchpole and M. B. King, Ind. Eng. Chem. Res., 1994, 33, 1828-1837.

82 C.-H. He and Y.-S. Yu, Ind. Eng. Chem. Res., 1998, 37, 3793-3798.

83 T. Funazukuri, C. Y. Kong and S. Kagei, Ind. End. Chem. Res., 2000, 39, 835-837.

84 A. Laesecke and C. D. Muzny, J. Phys. Chem. Ref. Data, 2017, 46, 013107. 